\title{
Un estudio sobre los materiales curriculares inipressos en Educación Fisica: implicaciones para la formación del profesorado
}

\author{
J. Pere Molina Alventosa* \\ Carmen Peiró Velert** \\ José Devis Devis*
}

\begin{abstract}
Resumen: En este artículo se presenta la fase descriptiva de una investigación sobre materiales curriculares impresos derivados del análisis de un cuestionario elaborado al efecto. El cuestionario se ha aplicado en forma de entrevista a una muestra representativa de profesores y profesoras de educación física de la Autonomía Valenciana que trabajan en la Enseñanza Secundaria Obligatoria. Se presentan los resultados generales relativos a la elección, la elaboración y el uso de los materiales. Posteriormente, se discuten estos resultados y comentan sus implicaciones para la formación del profesorado. Palabras clave: Materiales Curriculares, Educación Física, Pensamiento del Profesorado, España, Formación del Profesorado
\end{abstract}

Desde el mismo origen de la escuela, el profesorado se ha servido de distintos tipos de materiales curriculares como utensilios de trabajo para desarrollar su labor docente. La importancia de estos materiales se ha visto aumentada con la extensión de la escolarización de masas que han adoptado los países modernos desde el siglo XIX. Se trata de instrumentos u objetos que se utilizan en el contexto escolar como radiadores del proceso de enseñanza-aprendizaje con el fin de comunicar

"Departament d' Educació Física i Esportiva.

** Departament de Didáctica de la Expressió M., P. i Corporal. Universitat de Valencia España.

Movimento, Porto Alegre, v. 10, n. 1, p. 41-70,janeiro/abril de 2004 
contenidos o de favorecer, orientar o evaluar este proceso (Zabala, 1990; Gimeno, 1991; San Martín, 1991; Parcerisa, 1996). De entre ellos, los materiales impresos se han difundido ampliamente en las escuelas de los sistemas educativos contemporáneos, de tal manera que la mayor parte de las tareas y del tiempo escolar se desarrolla utilizando este tipo de materiales (Stodolsky, 1989; Westbury, 1990; Zahorik, 1991; Área, 1991; Johnsen, 1996). Son materiales que hacen referencia a textos, representaciones o adaptaciones de textos en combinación con iconos o imágenes (Área, 1994). Así pues, el texto es la manera esencial que tienen estos materiales de elaborar, almacenar y recuperar información, es decir, de tratar el conocimiento cultural y escolar, ya sea en formato de libro de texto o de documental de vídeo donde se representa y adapta un guión, es decir, un texto.

Sin embargo, este tipo de materiales son de reciente expansión en la educación física, en comparación con el resto de asignaturas escolares, ya que en esta asignatura han dominado los recursos o medios técnicos ligados a las actividades físicas, tales como pelotas o colchonetas. En el contexto español, la reforma educativa de la década de 1990 ha estimulado considerablemente la elaboración de materiales impresos en educación física y las editoriales se han dedicado desde entonces a publicar libros de texto de esta asignatura escolar para el alumnado de los distintos niveles educativos. Precisamente esta proliferación de los materiales impresos y la falta de estudios empíricos sobre su uso motivaron la investigación que ofrece el soporte empírico a este trabajo. Como comentamos en un artículo publicado en esta misma revista, en el que abordamos el marco teórico previo a la investigación, el profesorado es el centro de atención del estudio porque, según la literatura, es el colectivo de la comunidad educativa del que dependen las decisiones relativas a la selección, elaboración en su caso y forma de uso del material escrito en el contexto escolar (Devís et al., 2001). De ahí que algunos de los datos de la investigación y su discusión crítica sugieran diversas implicaciones para la formación del profesorado de educación física, el aspecto central de este trabajo. Pero antes comentaremos brevemente las principales líneas de investigación sobre materiales curriculares con la intención de contextualizar nuestro estudio.

Movimento, Porto Alegre, v. 10, n. 1, p. 41-70,janeiro/abril de 2004 


\section{La investigación sobre materiales curriculares impresos}

La gran difusión y uso escolar de los materiales impresos no se corresponde con la moderada atención dispensada por la investigación educativa, tal y como se desprende de las revisiones consultadas (Woodward, Elliot y Nagel, 1988; Weinbrenner, 1992; Área, 1994; Abraham y Rojas, 1997). Aún así, se observa la existencia de estudios realizados desde diferentes perspectivas teóricas y objetivos que pueden agruparse en cuatro grandes líneas de investigación centradas en: a) la eficacia de los materiales; b) el papel mediador del alumnado y el profesorado; c) el análisis del contenido del material; y d) el uso de materiales en contextos reales.

a) La eficacia de los materiales. $\mathrm{Al}$ amparo de las investigaciones proteso-producto dentro de la enseñanza surgieron diversas pesquisas de naturaleza correlacional o normativa que trataban de comparar la eficacia de distintos materiales y establecer relaciones empíricas demostrables. Se comparaba el uso de distintos materiales para encontrar el material más eficaz y buscaban identificar las características físicas de los materiales que mayor rendimiento producían en el aprendizaje, midiendo este último mediante tests estandarizados de rendimiento académico. Algunas investigaciones formaron parte de proyectos de desarrollo y cambio curricular y trataban de comprobar la fidelidad y correspondencia entre las metas de los programas y el proceso de aplicación de los nuevos materiales impresos (Fullan, 1983). Estas investigaciones recibieron muchas críticas porque tenían una visión instrumental de los materiales curriculares, se identificaron problemas en los diseños cuasiexperimentales y correlaciónales utilizados, y no arrojaron resultados concluyentes sobre qué materiales eran más efectivos (Área, 1991 y 1994). Además, creaban una especie de caja negra entre las variables del proceso y las variables del producto que ignoraban el papel que pudieran jugar los procesos intermedios.

b) El papel mediador del alumnado y el profesorado. Las críticas y las deficiencias conceptuales de los modelos del procesoproducto, junto con la implantación de teorías del

Movimento, Porto Alegre, v. 10, n. 1, p. 41-70,janeiro/abril de 2004 
aprendizaje de corte cognitivo y estrategias de procesamiento humano de la información, favorecen el desarrollo de modelos mediacionales centrados en el alumnado y el profesorado. Se trata de cambiar la visión conductista por una visión cognitivista que considere al profesor y a los estudiantes como individuos activos que captan y procesan información y que no sólo reaccionan de forma rutinaria, sino que también actúan tomando decisiones conscientes y viendo su acción como parte de una estructura significativa de interacción (Hanke, 1987). Es decir, se trata de arrojar algo de luz al interior de la caja negra, centrándose en los procesos mediacionales de los principales actores de la enseñanza. En relación con el alumnado, se realizaron investigaciones para encontrar relaciones entre las características instruccionales de los materiales y los procesos cognitivos de los estudiantes que permitieran conocer el nivel de asimilación, por parte del alumnado, de la información transmitida por los materiales. Los resultados obtenidos indicaron que los materiales y sus formas de presentación eran simples vehículos de información que no tenían un efecto real sobre el aprendizaje (Clark, 1983). En cuanto al papel mediador del profesorado, las pesquisas realizadas se centraron, especialmente, en el pensamiento del profesorado sobre los libros y materiales curriculares, realizadas mediante encuestas sobre las opiniones, intereses, percepciones y expectativas que le merece este tipo de materiales (p. ej. Connelly y Ben-Peretz, 1980; Ben-Peretz y Tamir, 1981; Marsh, 1984; Marsh et al, 1985; Shannon, 1983). Precisamente, las primeras investigaciones españolas que aparecieron sobre materiales impresos se ocuparon de estos aspectos del pensamiento del profesorado y surgieron en conexión con el proceso de institucionalización y expansión de la investigación educativa en el país (Tiana, 1998).

c) El análisis del contenido del material. Esta línea de investigación surge bajo la influencia de la 'nueva sociología de la educación' a finales de la década de 1970 y comienzos de la siguiente, destacando el análisis ideológico del conocimiento escolar contenido en los libros de texto (Apple, 1982 y 1989). En el contexto español, las primeras investigaciones de este tipo, realizadas a finales de los

Movimento, Porto Alegre, v. 10, n. 1, p. 41-70,janeiro/abril de 2004 
años ochenta, se centraron en el análisis de los libros de texto para desentrañar los mensajes ocultos relativos al racismo, el sexismo y otras manifestaciones ideológicas (p. ej. Calvo, 1989; Garretay Careaga, 1987; Moreno, 1987). Desde los noventa, el análisis del conocimiento escolar se aborda desde la óptica particular de distintas disciplinas escolares y se ocupa, tanto del sexismo como de la educación del desarrollo o la educación del ciudadano (p. ej. Argibay, Celorio y Celorio, 1990; Blanco, 2000; López y de la Caba, 2002). Algunas tesis doctorales analizan los libros de texto en relación con el modelo curricular de la reforma educativa española de la década de 1990 (Parcerisa, 1995; García Pascual, 1996) y sus repercusiones en la regulación de la profesionalidad docente (Cantarero, 2000). En esta línea de investigación se sitúan la mayoría de las investigaciones desarrolladas en la educación física sobre libros de texto y paquetes curriculares, ya sea para ocuparse del sexismo como de amplios análisis de los modelos curriculares, profesionalidad y relación con el trabajo del profesorado, tanto a nivel internacional (Brown, 1990; Dewar, 1985; Hildreth, 1981; Placek, 1989; Schempp, 1982) como español (Delgado, Barrera y Medina, 1992; Peiró y Devís, 1994; Ribas y Guerrero, 1994).

d) El uso de materiales en contextos reales. Durante la última década existen diversas voces que reclaman la necesidad de evaluar los libros y materiales dentro de los contextos curriculares y en situaciones de uso real (Área, 1991; Kirk, 1990; Santos, 1991; Venezky, 1992). El objetivo es conocer qué hace realmente el profesorado con los materiales curriculares durante el desarrollo de la enseñanza y con qué propósitos. Los estudios más recientes desde esta orientación de la investigación sobre materiales impresos, han aportado evidencia empírica de la influencia que ejercen otros factores como el contexto, las creencias del profesorado y las características de la materia en las formas de uso de este tipo de materiales (p. ej. Putnam, 1992; Remillard, 1992, 1999 y 2000). En España, Área (1986 y 1991) y Güemes (1994) han realizado estudios cualitativos sobre el papel que juegan los materiales impresos en la planificación, el proceso de instrucción, las decisiones del profesorado y las estructuras comunicativas de la clase.

Movimento, Porto Alegre, v. 10, n. 1, p. 41-70,janeiro/abril de 2004 
La pesquisa que proporciona los datos empíricos a este artículo combina, en dos fases, la línea de investigación mediacional del profesorado y la del uso de materiales en contextos reales. En la primera fase se trata de conocer lo que piensa y dice el profesorado de educación física de la Enseñanza Secundaria Obligatoria (ESO: 12-16 años) de la Autonomía Valenciana (España) sobre el uso de los materiales impresos a través de un cuestionario. Entre la información derivada del cuestionario se esperan obtener una serie de perfiles profesionales de uso de materiales que sirvan para identificar grupos de profesores y profesoras, de los que se seleccionen varios de cada grupo, para profundizar en las diversas circunstancias y características del uso real de los materiales en las clases, pero en esta ocasión mediante el estudio de casos con fuerte base observacional. Se trata de un diseño que combina una metodología cuantitativa, de la que se obtiene información general de una muestra relativamente amplia del profesorado, y otra metodología cualitativa que ofrece información rica y profunda sobre el uso de materiales en las clases de educación física de un pequeño grupo de profesores (ver Devís et al, 2001). A continuación detallamos los principales aspectos de la metodología cuantitativa que sirve a los propósitos de este artículo, mientras que se sigue desarrollando y analizando el estudio de caso cualitativo.

\section{Metodología}

La estrategia metodológica de la fase cuantitativa de nuestra investigación obedece a un diseño descriptivo, sustentado por una epistemología objetivista y derivado del análisis de un cuestionario elaborado al efecto.

\section{La muestra}

La muestra que se utilizó era representativa del profesorado de Educación Física en la ESO de la Comunidad Valenciana. Se definió a partir de datos poblacionales del curso escolar 1999-2000 proporcionados por la administración educativa (Servei de Formado del Professorat de la Conselleria de Cultura i Educado) de la Generalitat Valenciana. La muestra teórica señalaba los 300 sujetos para un error muestral de un $5 \%$. Además, se estratificó proporcionalmente según la provincia

$$
\text { (Alicante, }
$$

$$
\text { Valencia }
$$

Movimento, Porto Alegre, v. 10, n. 1, p. 41-70,janeiro/abril de 2004 
Castellón), la titularidad del centro (públicos frente a concertados y privados) y el tamaño de dichos centros (menos de 500 alumnos, entre 500 y 1000 , y más de 1000 alumnos). La muestra final a la que se administró el cuestionario fue de 310 profesores y profesoras que impartían ESO (210 hombres y 100 mujeres $)$ con edades comprendidas entre los 24 y 64 años de edad ( $m=37,7$; $d=8,7)$.

\section{Instrumento y procedimiento de recogida de datos}

Ante la falta de un cuestionario que atendiese a la finalidad concreta de nuestro estudio, elaboramos uno específico, cuyo proceso y características ya han sido descritas con detalle en otros trabajos (ver Molina et al. 2001a y Molina et al. 2001b). Las preguntas del cuestionario hacían referencia a variables demográficas o contextuales del profesorado y a una serie de variables de estudio agrupadas en las siguientes cuatro dimensiones: 1) elección de materiales; 2) materiales utilizados; 3) elaboración; y 4) formas de uso de materiales impresos. Las respuestas eran estructuradas y múltiples con alguna opción semiestructurada.

El cuestionario se aplicó a través de entrevista personal, cara a cara, porque así se conjugaba la máxima participación posible de la muestra seleccionada, la menor mortandad en las respuestas y la máxima calidad en los datos obtenidos. En la administración del cuestionario se contó con la colaboración de un equipo de entrevistadores a los que se preparó previamente. La duración media resultante por entrevista fue de 37 minutos.

La fiabilidad de este procedimiento de recogida de datos se garantizó a través de la estandarización en la administración del cuestionario, evitando diferencias en la forma de aplicarlo entre los distintos entrevistadores y entre un mismo entrevistador en sus distintas entrevistas. Para conseguirla se dedicó un periodo previo a la preparación del equipo de entrevistadores y se realizó un estudio piloto sobre 11 profesores y profesoras de ESO cuyo objetivo fue valorar el correcto funcionamiento del cuestionario en situación real y realizar los ajustes pertinentes para la versión definitiva.

En cuanto a la validez interna, la forma más potente que se puede aplicar a cuestionarios y guiones de entrevistas es la validez de contenido (Fox, 1981). En nuestro caso, ésta se atendió

Movimento, Porto Alegre, v. 10, n. 1, p. 41-70,janeiro/abril de 2004 
desde tres aspectos: a) la lógica del cuestionario, mediante su sistematización en dimensiones y categorías; b) la representatividad conceptual en relación con la literatura sobre el tema y con aportaciones de asesores de educación física de varios centros de profesores y; c) la revisión a través de un juicio de expertos y del estudio piloto.

\section{Variables de investigación y análisis de datos}

En nuestro estudio descriptivo distinguimos dos tipos de variables. Por una parte, las de carácter general referidas a los aspectos demográficos o contextuales que conforman la dimensión socioprofesional del profesorado (sexo, edad, titulación específica en educación física, situación laboral, titularidad del centro, etapa educativa de adscripción docente, participación en actividades de formación permanente, años de docencia en educación física, años de ejercicio en el centro). Por otra parte, disponemos de las variables de estudio específicamente relacionadas con cada una de las dimensiones antes señaladas. Para este trabajo en concreto, utilizaremos algunos resultados generales, sin cruzar con variables demográficas, que nos servirán para analizar críticamente y derivar implicaciones para la formación del profesorado. Estos resultados tienen que ver con las siguientes variables y categorías de variables, entendiéndose estas últimas como un agrupamiento de variables relacionadas con una misma idea:

La implicación del profesorado en la elección de materiales impresos. Esta variable se configura a partir de la pregunta del cuestionario: todos los materiales impresos que utilizas ¿los has elegido tú? La escala de respuesta es: $0=$ no, ninguno; $1=$ algunos; $2-l a$ mayoría-, 3- sí, todos.

Los criterios utilizados en la elección de materiales impresos y su grado de importancia viene representados por un grupo de variables que pueden verse en la figura 2. Cada una de las variables se responde de acuerdo a la escala siguiente: $0=$ ninguna importancia; $1=$ poca importancia; $2=$ bastante importancia; $3=$ mucha importancia.

Movimento, Porto Alegre, v. 10, n. 1, p. 41-70,janeiro/abril de 2004 
El tipo de materiales impresos utilizados. Esta categoría se define a partir de señalar si son (o no) utilizados una serie de materiales impresos para el profesorado, para el alumnado, para el centro y para la familia (ver las tablas).

La frecuencia de uso de los materiales impresos utilizados. Esta categoría se define a partir de indicar la frecuencia de uso de cada uno de los materiales señalados como utilizados en la siguiente escala de respuesta $0=$ nunca; $1=$ alguna vez; $2=$ pocas veces; $3=$ bastante; $4=$ mиу а menudo.

Los responsables de la elaboración de los materiales impresos utilizados. Esta categoría se refiere a una pregunta con respuesta múltiple, es decir, que permite señalar más de una opción de las tres variables presentadas (ver tabla 2).

Las formas de elaboración de los materiales por parte del profesorado. También se refiere a una pregunta con respuesta múltiple a tres variables posibles (ver tabla 3 ).

Las razones por las que el profesorado elabora los materiales utilizados y su grado de importancia. Esta categoría la forman diversas variables que pueden observarse en la figura 3. La escala de respuesta es: $0=$ ninguna importancia; $1=$ poca importancia; $2=$ bastante importancia; $3=$ mucha importancia.

El análisis de datos se realizó mediante el paquete estadístico SPSS v.10.0 para Windows. Se utilizaron índices estadísticos para describir las variables obtenidas y, en su caso, para establecer la magnitud y significación de las relaciones entre las mismas. En este artículo sólo presentamos los estadísticos descriptivos, referidos a medias y porcentajes, sobre las variables de estudio anteriormente mencionadas.

\section{Resultados}

En este apartado se presentan los resultados descriptivos correspondientes a las variables de estudio señaladas y que hacen referencia a: 1) la elección de los materiales impresos; 2) la elaboración; y 3) la utilización y frecuencia de uso de los materiales impresos.

Movimento, Porto Alegre, v. 10, n. 1, p. 41-70,janeiro/abril de 2004 


\section{La elección de los materiales impresos}

La implicación del profesorado de educación física en la elección de los materiales impresos que utiliza es muy grande, tal y como representa la figura 1 . De los 310 profesores y profesoras entrevistadas, la mayor parte del profesorado, el 94,8\%, elige todos o la mayoría de los materiales que utiliza. Sólo un 5,2\% elige alguno o ninguno de los materiales que utiliza.

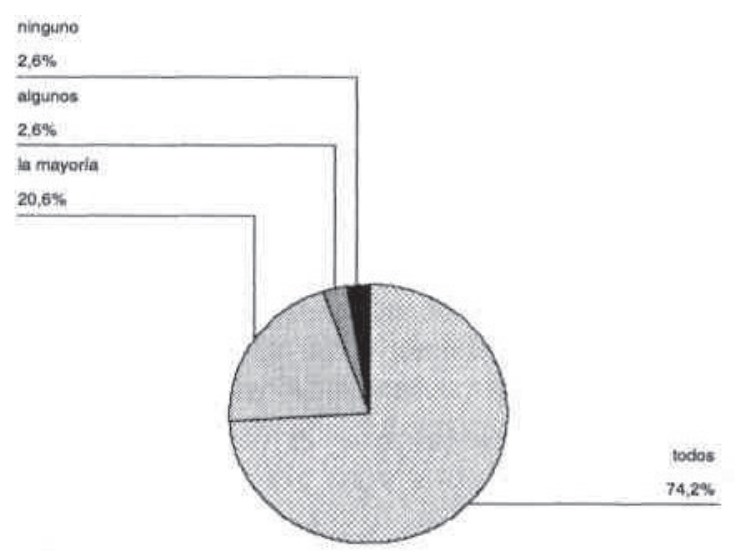

Figura 1. Implicación del profesorado en la elección de los materiales que utiliza

Entre los criterios o variables utilizadas por el profesorado en la elección de materiales, la figura 2 muestra los promedios de respuesta relativos a su importancia. Los criterios considerados más importantes por el profesorado son, dentro de una escala de 0 a 4 , la calidad de los contenidos del material $(\mathrm{m}-2,72)$ y la adecuación al alumnado $(\mathrm{m}=$ $2,68)$. La contribución del material a la formación permanente $(\mathrm{m}=$ $2,41)$, la correspondencia con la ideología del profesor o profesora (m $=2,19)$ y la adecuación al curriculum oficial $(\mathrm{m}=2,17)$ son los criterios que le siguen en importancia. Por detrás queda el bajo coste del material $(\mathrm{m}=1,75)$ y su presentación $(\mathrm{m}=1,73)$. Los criterios o variables menos importantes son la correspondencia del material con la ideología del centro $(\mathrm{m}=1,37)$ y el que estén escritos en valenciano $^{1}(\mathrm{~m}=1,07)$.

1 Valenciano es el nombre que recibe el idiomacatalán que se habla en la Autonomía Valenciana.

Movimento, Porto Alegre, v. 10, n. 1, p. 41-70,janeiro/abril de 2004 




Figura 2. Promedio de importancia de los criterios empleados por el profesorado en la elección $(\mathrm{n}>309)$

\section{La elaboración de los materiales impresos}

Los resaltados sobre la elaboración de materiales, presentados en las tablas 1 y 2 , deben interpretarse con precaución porque el número de profesores y profesoras que contesta a cada tipo material es distinto. Además hay que recordar que la opción de respuesta para cada tipo de material es múltiple. Teniendo todo esto en cuenta, y a partir de los resultados reflejados en la tabla 2, la mayoría del profesorado considera que las editoriales son las responsables de elaborar los libros de texto de uso propio $(96,4 \% \mathrm{n}=139)$ y para el alumnado $(98,6 \%, \mathrm{n}=72)$. También obtienen altos porcentajes de respuesta las fuentes informativas, documentales o de consulta del profesorado $(82,4 \%, \mathrm{n}=279)$ y del alumnado $(76,9 \%, \mathrm{n}=251)$.

Por otra parte, la implicación del profesorado en la elaboración de sus materiales es bastante relevante. Todo el profesorado entrevistado elabora algún tipo de material impreso que utiliza en sus clases a lo largo del curso. En la elaboración de estos materiales, predomina la labor individual sobre el trabajo en equipo. El tipo de material que más se elabora individualmente son los destinados a las fichas de sesiones $(78,6 \%, \mathrm{n}=224)$ y el registro de datos $(69,7 \%, \mathrm{n}=$ 297), y el más elaborado en equipo son las secuencias de contenido y programaciones $(43,8 \%, n=265)$. También destaca la implicación del profesorado en la elaboración de proyectos y memorias de área para el centro. Precisamente en este tipo de materiales es donde encontramos mayor elaboración en equipo $(56,3 \%, n=268)$ que individual $(46,3 \%$, $\mathrm{n}=268)$.

Movimento, Porto Alegre, v. 10, n. 1, p. 41-70,janeiro/abril de 2004 
Tabla 1. Responsables de la elaboración de los materiales utilizados en porcentajes

\begin{tabular}{|c|c|c|c|c|c|}
\hline & & & & & \\
\hline & & \multirow{2}{*}{$n^{*}$} & \multirow{2}{*}{$\%$ Editoriales } & \multicolumn{2}{|c|}{$\begin{array}{c}\% \text { elaboración propia } \\
\text { profes. }\end{array}$} \\
\hline & & & & individual & en grupo \\
\hline \multirow{10}{*}{ 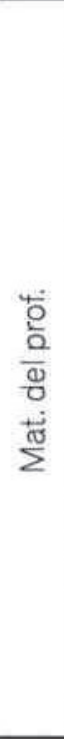 } & $\begin{array}{l}\text { Fuentes informativas, } \\
\text { documentales o de } \\
\text { consulta }\end{array}$ & 279 & 82,4 & 37,3 & 22,9 \\
\hline & $\begin{array}{l}\text { Libro de texto del } \\
\text { profesor }\end{array}$ & 139 & 96,4 & 2,9 & 4,3 \\
\hline & $\begin{array}{l}\text { Secuencias de } \\
\text { contenidos y } \\
\text { programaciones }\end{array}$ & 265 & 20,4 & 57,4 & 43,8 \\
\hline & Unidades didácticas o & & & & \\
\hline & libros de unidades & 250 & 33,6 & 68,4 & 36,8 \\
\hline & didácticas & & & & \\
\hline & Fichas de sesiones & 224 & 15,6 & 78,6 & 25 \\
\hline & $\begin{array}{l}\text { Recopilaciones de } \\
\text { ejercicios, juegos y otras } \\
\text { A.F. }\end{array}$ & 234 & 42,7 & 56 & 31,2 \\
\hline & $\begin{array}{l}\text { Materiales para el } \\
\text { registro de datos }\end{array}$ & 297 & 10,1 & 69,7 & 25,6 \\
\hline & Otros & 27 & 40,7 & 51.9 & 0 \\
\hline \multirow[t]{6}{*}{$\Sigma$} & $\begin{array}{l}\text { Fuentes informativas, } \\
\text { documentales o de } \\
\text { consulta }\end{array}$ & 251 & 76,9 & 38,2 & 22,3 \\
\hline & Libros de texto & 72 & 98,6 & 2,8 & 1,4 \\
\hline & $\begin{array}{l}\text { Cuadernos de } \\
\text { actividades y fichas de } \\
\text { trabajo }\end{array}$ & 181 & 28,7 & 49,7 & 24,9 \\
\hline & $\begin{array}{l}\text { Recopilaciones de } \\
\text { ejercicios, juegos y otras } \\
\text { A.F. }\end{array}$ & 152 & 21,1 & 46,1 & 34,2 \\
\hline & Diarios de clase & 93 & 2,2 & 41.9 & 18,3 \\
\hline & Otros & 54 & 24,1 & 46,3 & 18,5 \\
\hline \multicolumn{2}{|c|}{$\begin{array}{l}\text { Documentos informativos } \\
\text { para la familia }\end{array}$} & 108 & 1,9 & 58,3 & 37 \\
\hline \multicolumn{2}{|c|}{$\begin{array}{l}\text { Proyectos y memorias de área } \\
\text { para el centro }\end{array}$} & 268 & 4,1 & 46,3 & 56,3 \\
\hline \multicolumn{2}{|c|}{ Otros } & 6 & 0 & 50 & 16,7 \\
\hline
\end{tabular}

Movimento, Porto Alegre, v. 10, n. 1, p. 41-70,janeiro/abril de 2004 
Tabla2. Formas de elaboración del material por parte del profesorado

\begin{tabular}{|c|c|c|c|c|c|}
\hline & & $n^{\star}$ & $\begin{array}{l}\% \text { Recopilar } \\
\text { y organizar }\end{array}$ & $\begin{array}{c}\% \\
\begin{array}{c}\text { Modificar y } \\
\text { adaptar }\end{array}\end{array}$ & $\begin{array}{c}\% \\
\text { Crear } \\
\text { nuevos }\end{array}$ \\
\hline \multirow{8}{*}{ 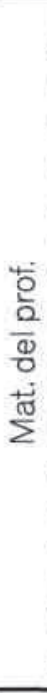 } & $\begin{array}{l}\text { Fuentes informativas, } \\
\text { documentales o de } \\
\text { consulta }\end{array}$ & 138 & 52,9 & 71 & 41,3 \\
\hline & $\begin{array}{l}\text { Libro de texto del } \\
\text { profesor }\end{array}$ & 9 & 44,4 & 44,4 & 44,4 \\
\hline & $\begin{array}{l}\text { Secuencias de } \\
\text { contenidos y } \\
\text { programaciones }\end{array}$ & 241 & 40,2 & 82,6 & 43,6 \\
\hline & $\begin{array}{l}\text { Unidades didácticas o } \\
\text { libros de unidades } \\
\text { didácticas }\end{array}$ & 230 & 37 & 82,6 & 53 \\
\hline & Fichas dé sesiones & 218 & 30,3 & 67,4 & 63.8 \\
\hline & $\begin{array}{l}\text { Recopilaćiones de } \\
\text { ejercicios, juegos y otras } \\
\text { A.F. }\end{array}$ & 188 & 52,7 & 69,7 & 45,2 \\
\hline & $\begin{array}{l}\text { Materiales para el } \\
\text { registro de datos }\end{array}$ & 252 & 26,2 & 48,4 & 62,3 \\
\hline & Otros & 14 & 28,6 & 35,7 & 57,1 \\
\hline \multirow{9}{*}{ 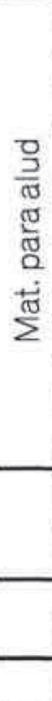 } & $\begin{array}{l}\text { Fuentes informativas, } \\
\text { documentales o de } \\
\text { consulta }\end{array}$ & 129 & 55,8 & 66,7 & 49,6 \\
\hline & Libros de texto & 3 & 66,7 & 33,3 & 0 \\
\hline & $\begin{array}{l}\text { Cuadernos de } \\
\text { actividades y fichas de } \\
\text { trabajo }\end{array}$ & 121 & 33,1 & 56,2 & 55.4 \\
\hline & $\begin{array}{l}\text { Recopilaciones de } \\
\text { ejercicios, juegos y otras } \\
\text { A.F. }\end{array}$ & 107 & 49,5 & 63,2 & 46,2 \\
\hline & Diarios de clase & 52 & 15,4 & 30,8 & 73,1 \\
\hline & Otros & 31 & 32,3 & 45,2 & 54,8 \\
\hline & $\begin{array}{l}\text { Documentos } \\
\text { informativos para la } \\
\text { familia }\end{array}$ & 96 & 19,8 & 35,4 & 64,6 \\
\hline & $\begin{array}{l}\text { Proyectos y memorias de } \\
\text { área para el centro }\end{array}$ & 241 & 31,5 & 66,4 & 41,9 \\
\hline & Otros & 4 & 50 & 75 & 25 \\
\hline
\end{tabular}

${ }^{\star}{ }^{*} \mathrm{n}=$ número de respuestas obtenidas $\mathrm{y}$ válidas en el análisis

Movimento, Porto Alegre, v. 10, n. 1, p. 41-70,janeiro/abril de 2004 
Los resultados relativos a las formas de elaboración de materiales se observan en la tabla 3. En la tabla se observa que el profesorado se dedica, principalmente, a la 'modificación y adaptación' de materiales existentes. Un alto porcentaje del profesorado modifica y adapta las unidades didácticas o libros de unidades didácticas $(82,6 \%, \mathrm{n}=241)$ y las secuencias de contenidos y las programaciones $(82,6 \%, \mathrm{n}=230)$. En un porcentaje menor, el profesorado modifica y adapta las fuentes informativas, documentales y de consulta $(66,7 \%, \mathrm{n}=129)$, así como las recopilaciones de ejercicios dirigidas al alumnado $(63,2 \%, \mathrm{n}=107)$, y los proyectos y memorias de área para el centro escolar $(66,4 \%, n=241)$

La 'recopilación y organización' que realiza el profesorado en los diferentes materiales arroja porcentajes inferiores a la 'modificación y adaptación' y también es inferior el número de profesores y profesoras que contesta. Así, por ejemplo, el porcentaje más alto $(66,7 \%)$ se refiere al libro de texto para el alumnado, sin embargo el número de respuestas desde las que se obtiene este porcentaje es muy bajo $(\mathrm{n}=$ 3 ). Las fuentes informativas, tanto para el profesorado como para el alumnado $(52,9 \%, \mathrm{n}=138 ; 55,8 \%, \mathrm{n}=129)$, y la recopilación de ejercicios, también para el profesorado y el alumnado $(52,7 \%, \mathrm{n}=$ $188 ; 49,5 \%, \mathrm{n}=107)$, reciben los porcentajes más altos.

El profesorado que dice dedicarse a la 'creación de nuevos materiales' lo hace en fichas de sesiones $(63,8 \%, \mathrm{n}=218)$, materiales de registro de datos $(62,3 \%, \mathrm{n}=252)$ y unidades didácticas $(53 \%, \mathrm{n}=$ 230). Los documentos informativos para la familia también recibe un porcentaje considerable $(64,6 \%)$, si bien sobre un menor número de entrevistados $(\mathrm{n}=96)$.

Finalmente, en la figura 3 puede verse el grado de importancia que asigna el profesorado a las razones de la elaboración de materiales impresos, dentro de una escala de 0 a 3 . Debemos señalar que todo el profesorado manifestó realizar algún tipo de elaboración por lo que el número de respuestas en cada una de las razones presentadas coincidió con la muestra total, a excepción de una donde se perdió un caso. Las cuatro razones más importantes son la mejor adaptación de éstos a su alumnado $(\mathrm{m}=2,56)$, la comodidad de ser materiales propios $(\mathrm{m}=2,43)$, su contribución a la formación permanente $(\mathrm{m}=2,28)$ y la correspondencia con su ideología $(\mathrm{m}=$ $2,01)$. El profesorado considera menos importante que el material publicado no responda a sus intereses $(\mathrm{m}=1,85)$, el

Movimento, Porto Alegre, v. 10, n. 1, p. 41-70,janeiro/abril de 2004 


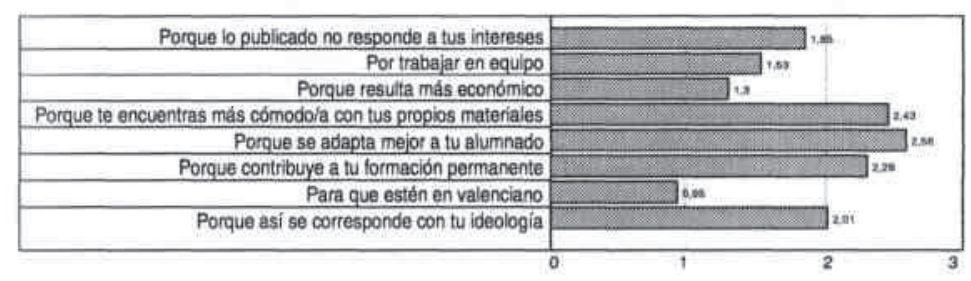

Figura 3. Promedio obtenido en las razones de elaboración de materiales por el profesorado $(\mathrm{n}>309)$

trabajo en equipo $(\mathrm{m}=1,53)$ y que el material resulte más económico $(\mathrm{m}=1,3)$. La razón que menos importancia le asigna el profesorado a la hora de elaborar materiales es para que estén en valenciano $(\mathrm{m}=$ $0,95)$.

\section{La utilización y la frecuencia de uso de los materiales}

La tabla 3 refleja los resultados concernientes al tipo de materiales utilizados por el profesorado, tanto los de uso propio, los que utiliza para el alumnado, así como los que utiliza para la familia y para el centro escolar. La tabla se completa con la frecuencia de uso de estos materiales.

En términos generales, el profesorado indica que utiliza mucho más los materiales propios que los dirigidos al alumnado, el centro o la familia. Entre los materiales del profesorado, el más destacado es el dedicado al registro de datos que declara utilizarlo el $95,8 \%$ de los profesores y profesoras, siendo su promedio de frecuencia de uso de 3,36 , es decir, 'bastante' según la escala. El $90 \%$ del profesorado también utiliza las fuentes informativas, aunque 'pocas veces' $(\mathrm{m}=$ 2,26 ) es su frecuencia de uso. Las secuencias de contenidos y las unidades didácticas también las utilizan gran parte del profesorado, el $85,5 \%$ y el $80,6 \%$ respectivamente, con frecuencias de uso cercanas al 'bastante' de la escala. El libro de texto es un material que utilizan pocos profesores para uso propio $(45,2 \%)$ y 'alguna vez' es su frecuencia de uso $(\mathrm{m}=1,24)$. Este material es el único que no muestra diferencias significativas entre el uso y el no uso.

Movimento, Porto Alegre, v. 10, n. 1, p. 41-70,janeiro/abril de 2004 
Tabla 3. Utilización y frecuencia de uso de materiales impresos

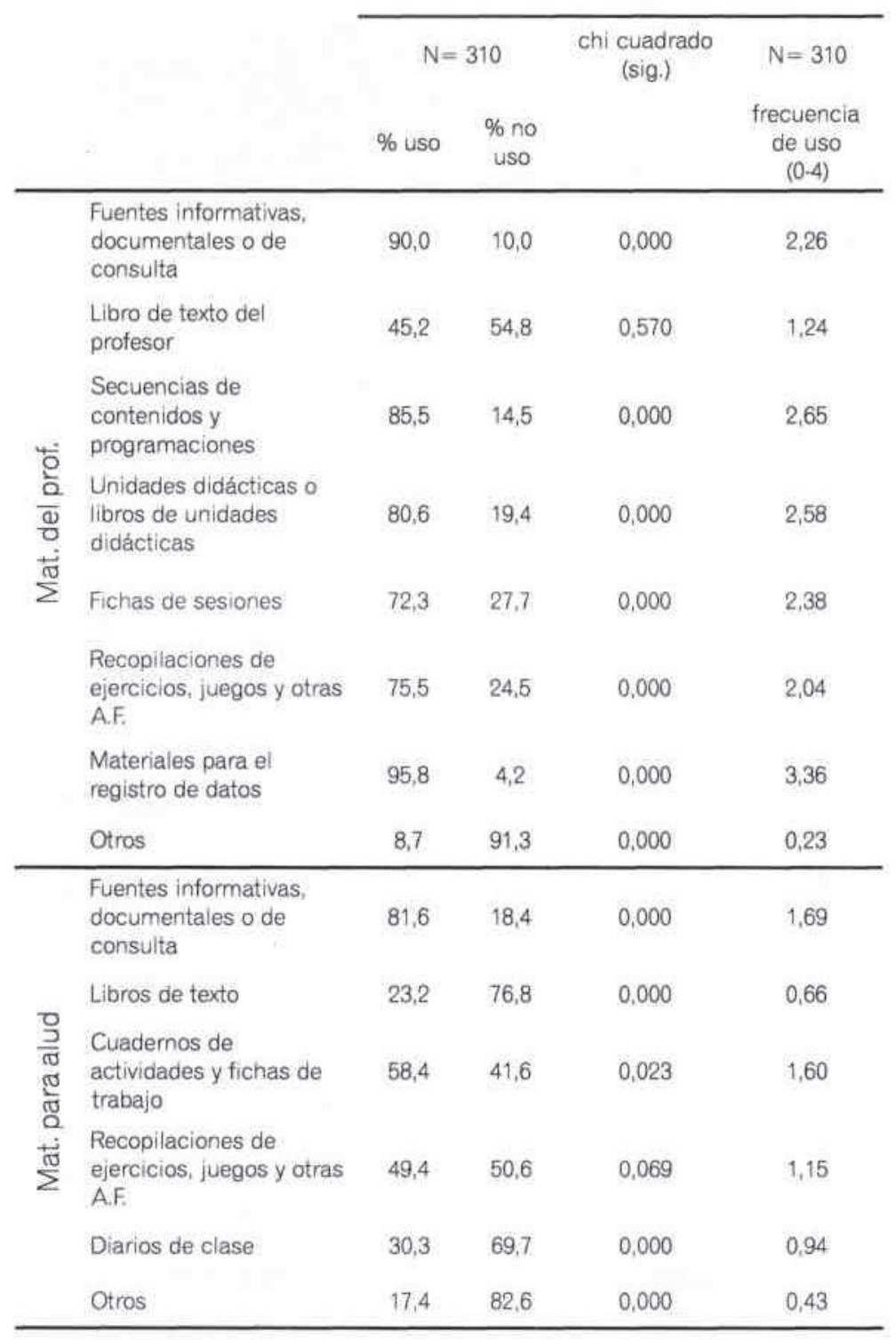

Movimento, Porto Alegre, v. 10, n. 1, p. 41-70,janeiro/abril de 2004 


\begin{tabular}{lcccc} 
(Continuación) & & & & \\
\hline $\begin{array}{l}\text { Documentos informativos } \\
\text { para familia }\end{array}$ & 35,2 & 64,8 & 0,000 & 0,60 \\
\hline $\begin{array}{l}\text { Proyectos y memorias de } \\
\text { área para el centro }\end{array}$ & 88,4 & 11,6 & 0,000 & 1,68 \\
\hline Otros & 1,9 & 98,1 & 0,000 & 0,02 \\
\hline
\end{tabular}

En cuanto a los materiales para el alumnado, el más utilizado por el profesorado es el relativo a las fuentes informativas $(81,6 \%)$, aunque con una frecuencia de uso cercana al 'pocas veces' $(\mathrm{m}=1,96)$ en la escala de nuestro estudio. Un material poco utilizado por el profesorado es el libro de texto $(23,2 \%)$, además con una frecuencia muy baja $(\mathrm{m}=0,66)$.

Los proyectos y memorias para el centro escolar lo utilizan muchos profesores y profesoras $(88,4 \%)$, pero con una frecuencia de uso baja que se sitúa cercana al 'pocas veces' $(\mathrm{m}=1,68)$ de la escala. Los materiales dirigidos a las familias tienen un uso testimonial.

\section{Discusión e implicaciones para la formación del profesorado}

El estudio que hemos presentado ofrece un conocimiento valioso relativo a cómo piensa y entiende el profesorado de educación física de la Autonomía Valenciana (España) la elección, la elaboración y el uso de materiales curriculares impresos en su actividad docente de la ESO. De esta manera se pueden tomar decisiones que puedan repercutir en el cambio o reforzamiento de algunos pensamientos y acciones con las que se supone que están conectados los primeros y, así, contribuir a la mejora profesional de los docentes. En este sentido, la formación del profesorado, tanto inicial como permanente, se convierte en un instrumento bisagra fundamental en la comunicación con los docentes en activo y los estudiantes que serán futuros profesores y profesoras de educación física. Por ello, en este apartado discutiremos críticamente los resultados anteriores y comentaremos algunas implicaciones que pueden tener en la formación de este colectivo profesional.

Movimento, Porto Alegre, v. 10, n. 1, p. 41-70,janeiro/abril de 2004 


\section{Sobre la elección de los materiales}

Los resultados de nuestro estudio reflejan que la gran mayoría del profesorado de secundaria entrevistado (94,8\%) elige los materiales que utiliza en su práctica docente, ya sea a nivel de uso personal como de uso con el centro, el alumnado o la familia. Este dato es, en sí mismo, revelador porque, contrariamente a lo que ocurre en otros lugares (Apple, 1984; Marsh et al., 1985), los departamentos, los centros educativos y, en ocasiones, la administración no juega un papel decisivo en la elección de los materiales. Además, la poca importancia que concede el profesorado a la ideología del centro como criterio de elección viene a apoyar esta idea. Si también tenemos en cuenta que manejan criterios de elección y entre ellos destacan la calidad de los contenidos del material impreso, así como la adecuación del mismo al alumnado, podemos pensar que el profesorado de educación física goza de una buena autonomía profesional. Sin embargo, un análisis crítico de los resultados nos invita a ser más cautelosos con este dato y derivar una serie de implicaciones acordes con dicho análisis.

Si bien los resultados indican que una gran mayoría del profesorado elige sus materiales, no podemos limitar la autonomía profesional a la elección de éstos o considerar que la elección es un aspecto decisivo de la misma. La elección de los materiales puede llegar a coartar la autonomía profesional del profesorado si, a la hora de elegir, opta por materiales impresos cerrados que deciden por él a nivel docente y le ahorran tiempo para emplearlo en otras tareas laborales, tal y como sugiere el estudio de Loewenberg y FeinamNemser (1988), centrado en las prácticas de estudiantes de profesorado.

No se trata de restarle importancia a la elección de los materiales, sin embargo hemos de reconocer que la elaboración propia de éstos es un indicador más ajustado a lo que entendemos por autonomía profesional o, al menos, un indicador de un nivel mayor de autonomía (Martínez Bonafé, 1998). Algunos autores se refieren a que la elección y la elaboración se encuentran en los extremos de un contínuum de posibilidades de participación del profesorado y, en definitiva, de autonomía profesional, donde el extremo de la elección es el más mecánico y menos participativo y el extremo de la elaboración es el más creativo y participativo (Devís y Peiró, 2004).

Movimento, Porto Alegre, v. 10, n. 1, p. 41-70,janeiro/abril de 2004 
En lo referente a los criterios que el profesorado considera más importantes a la hora de elegir los materiales, la 'calidad de los contenidos' y la 'adecuación al alumnado' surgen como los más destacados. No obstante, el concepto 'calidad' puede resultar problemático, puesto que dependiendo de cómo lo entienda e interprete cada profesor la elección de uno u otro material puede variar considerablemente. Así, por ejemplo, para unos la calidad puede tener que ver con un material que mantenga al alumnado haciendo ejercicio el mayor tiempo posible, mientras que para otros tiene más que ver con la vivencia del alumnado, la interacción y el ambiente de clase, así como lo que se transmite explícita e implícitamente (Tinning, 1992).

Por otra parte, los datos procedentes del cuestionario no responden a una pregunta clave para la formación del profesorado: ¿elige bien el profesorado de educación física sus materiales curriculares impresos? Esperamos contestar esta pregunta con la fase relativa al estudio de casos cualitativos donde la información más detallada complete las lagunas del cuestionario. En cualquier caso, la elección corre el peligro de realizarse teniendo en cuenta meras preferencias, visiones simplistas de lo que es divertido y significativo $o$ en nociones estereotipadas de lo que necesita y puede aprender el alumnado (Anyon, 1981; Loewenberg y Feiman-Nemser, 1988). Es decir, en última instancia, los criterios de la elección quedan a merced de la influencia de las distintas tendencias profesionales existentes y, por tanto, es previsible que en la elección se reproduzcan también las visiones socialmente dominantes dentro de la cultura profesional del colectivo. Además, una mayor implicación del profesorado en la elección no presupone una mejor elección de los materiales impresos. El alto porcentaje de profesorado que elige materiales impresos puede resultar influido por la ideología y las formas de enfocar y desarrollar el curriculum sugerido en los materiales (Apple, 1982 y 1989; Martínez Bonafé, 1998).

Dado que la elección de los materiales puede resultar problemática, consideramos que la mejora y el cambio del status quo del uso y elección de los materiales en la educación física y, en definitiva, en el desarrollo de la enseñanza de esta asignatura, se conseguirá mediante la construcción de estrategias que redunden en la formación del profesorado. Entre ellas destaca la formación en el análisis y evaluación de los materiales impresos para que el profesorado, especialmente el novel, llegue a emitir juicios de valor sobre los materiales y pueda tomar

Movimento, Porto Alegre, v. 10, n. 1, p. 41-70,janeiro/abril de 2004 
decisiones informadas en futuras situaciones de elección a partir de una serie de criterios coherentes (Ben-Peretz, 1988; Parcerisa, 1996, Remillard, 2000).

\section{Sobre la elaboración de materiales}

El profesorado de nuestro estudio asigna a las editoriales la responsabilidad de elaborar los libros de texto y las fuentes informativas, tanto para uso propio como para el alumnado, mientras que en la elaboración del resto de materiales es el profesorado el que se ve implicado mayoritariamente. En este reparto de responsabilidades las editoriales adoptan el papel de expertos en la elaboración de materiales relativos a contenidos principalmente teóricos y el profesorado asume el papel de experto en la elaboración de materiales de contenido más práctico, estimulando así la separación entre la teoría y la práctica en la educación física. Dado que lo deseable es que los materiales conjuguen, de manera coherente, tanto los conceptos teóricos (saber qué) con los procedimientos (saber cómo) y las actitudes (saber ser), la formación del profesorado debería ofrecer la oportunidad a los estudiantes de elaborar materiales de 'conexión teoría-práctica', tal y como los denominan Devís et al. (2001), de manera que el conocimiento teórico se experimente, aprenda y vincule a la práctica física. Así, es más probable que se facilite un aprendizaje experiencial más significativo y enriquecedor y una mejor asimilación e integración de los conocimientos (Devís y Peiró, 2004).

Por otra parte, nuestro estudio revela que el profesorado se dedica fundamentalmente a 'modificar y adaptar' materiales ya existentes, especialmente unidades didácticas, secuencias de contenidos, fuentes informativas y proyectos de área. En menor medida se dedica a 'crear nuevos' materiales, dirigiéndose fundamentalmente a fichas de sesiones, materiales de registro de datos y unidades didácticas. Los materiales que el profesorado dice 'recopilar y organizar' son, principalmente, fuentes informativas y compendios de ejercicios, juegos y actividades físicas. Como puede observarse en la tabla 2 , el profesorado presta más atención a materiales que le sirven a la planificación y desarrollo curricular en la práctica. Esta característica es coherente con una de las razones más importantes por las cuales el profesorado de nuestro estudio decide elaborar materiales, aquélla que contribuye a su formación permanente.

Movimento, Porto Alegre, v. 10, n. 1, p. 41-70,janeiro/abril de 2004 
En términos generales, estos resultados sugieren la existencia de una cierta autonomía del profesorado en la elaboración de materiales impresos. La intervención del profesorado en las tareas de modificación y evaluación del material supone tener que tomar decisiones y realizar adaptaciones atendiendo a las necesidades contextuales y particulares del alumnado en las que el material se aplicará. Así lo expresa la mayoría del profesorado de nuestro estudio al señalar, entre las razones que le mueven a elaborar materiales, que los elabora porque se adaptan mejor al alumnado.

Sin embargo, los distintos niveles de elaboración (recopilación, modificación y creación) implican y comprometen de forma diferente al profesorado e indican niveles diferentes de autonomía profesional. En este sentido, la formación inicial (y permanente) del profesorado debería proporcionar experiencias y ambientes de aprendizaje que inviten a la resolución de problemas, la toma de decisiones y la emergencia de habilidades referidas a la elaboración de materiales curriculares, dado que los estudiantes carecen del conocimiento y la experiencia necesaria para desarrollar el curriculum (Loewenberg y Feiman-Nemser, 1988). Tal vez resultaría conveniente, dada su complejidad, que los primeros cursos se centraran en la adquisición de experiencias, conocimiento, criterios y habilidades relativas al análisis de materiales curriculares, de tal manera que les capacitara para, en los últimos cursos, dedicarse a las tareas de elaboración. Tareas que irían desde la modificación de materiales ya existentes a la elaboración de nuevos materiales curriculares, con la posibilidad de experimentarlos y evaluarlos en la práctica durante el periodo del practicum que se realiza en los centros escolares.

Otro de los datos procedentes de nuestro estudio revela que la elaboración de los materiales curriculares impresos por parte del profesorado se lleva a cabo mayoritariamente de manera individual, con un escaso trabajo en grupo. Únicamente se evidencia un trabajo en colaboración del profesorado cuando se trata de proyectos y memorias para el centro escolar propiciado, tal vez, por la tradición institucional de elaborar las memorias por ciclos o departamentos, lo cual requiere el trabajo conjunto entre sus componentes.

Si bien este estudio no indaga las razones por las cuales el profesorado tiende a elaborar los materiales individualmente, este dato puede hacernos pensar la falta de cultura colaborativa

Movimento, Porto Alegre, v. 10, n. 1, p. 41-70,janeiro/abril de 2004 
existente tanto en los programas de formación inicial del profesorado como en la política educativa de la administración pública pertinente. Como señala Fraile (2000), este individualismo reinante acaba por obstaculizar el desarrollo profesional y la elaboración de materiales. En cambio, un énfasis en la colaboración y el conocimiento compartido evita el aislamiento del profesorado y reduce los obstáculos que impiden el enriquecimiento, la mejora profesional y la elaboración de materiales impresos (Devís, 1996; Nias, 1987; Peiró y Devís, 1995). Los programas deberían ofrecer a los estudiantes la oportunidad de participar en situaciones en las que tengan que trabajar en colaboración con sus compañeras y compañeros, tanto en la construcción del conocimiento de tipo más teórico como, por ejemplo, en la elaboración de materiales curriculares de posible aplicación a la realidad docente o de situaciones de enseñanza (Peiró, 2001).

\section{Sobre la utilización y frecuencia de uso del material}

El predominio de los materiales de uso propio del profesorado sobre los dirigidos al alumnado, el centro o la familia, refleja la importancia que el profesorado asigna a la planificación y el control de la enseñanza por encima del desarrollo de la misma. Si, además, los materiales para el registro de datos, secuencias de contenido y unidades didácticas son los que utiliza la mayoría del profesorado y con una frecuencia considerable, se apoya con mayor firmeza esa idea puesto que dichos materiales suelen tener como objetivos el control y la evaluación. La menor utilización y frecuencia de uso del material dirigido al alumnado indica que el profesorado presta menos atención al desarrollo de la enseñanza en comparación con la planificación y el control. Esto sugiere la necesidad de favorecer el uso (y la elaboración) de los materiales dirigidos al alumnado, los principales protagonistas de la enseñanza, hacia el que deben dirigirse todos los esfuerzos del profesorado. Para ello, se requiere estimular la participación del profesorado en proyectos e iniciativas de innovación y desarrollo curricular como estrategia fundamental en la formación inicial, principalmente a través del practicum, y en la formación permanente del profesorado por su conexión directa con la elaboración, uso y evaluación de materiales curriculares (Ben-Peretz, 1988).

Movimento, Porto Alegre, v. 10, n. 1, p. 41-70,janeiro/abril de 2004 
Un dato importante de la utilización de materiales se refiere al escaso uso del libro de texto para el alumnado, un $23,2 \%$, lo que indica que no está fuertemente implantado en la educación física. Este dato contrasta claramente con el de otros estudios que elevan mucho este porcentaje cuando se refieren al profesorado de otras asignaturas escolares. Así, por ejemplo, el estudio realizado por Zahorik (1991) con profesorado de niveles educativos y materias diferentes, eleva la cifra de uso al $82 \%$. En la educación física, los libros de texto poseen una imagen y presentación atrayente y original, pero su forma tradicional de uso usurpa la capacidad profesional del profesorado, puesto que deciden por él los contenidos y partes a tratar en la asignatura y lo reducen a simple aplicador práctico y continuador de las sesiones desarrolladas en sus páginas. Sin embargo, esto no quiere decir que desdeñemos las posibilidades del libro de texto, puesto que puede utilizarse de manera no convencional, es decir, como elemento de apoyo, consulta, o bien puede recurrirse a él para utilizar una parte concreta, unas páginas en particular o algunas de las ilustraciones que contenga para plantear o completar las actividades de clase. Esta función del libro no requiere que cada alumna o alumno tenga el suyo propio, sino que puede encontrarse en la biblioteca del aula o del centro y, además, puede facilitar un clima cooperativo en clase (Devís y Peiró, 2004).

Finalmente, merece la pena destacar la escasa utilización y frecuencia de uso de los materiales curriculares que tratan de favorecer el vínculo familia-escuela (en este caso, asignatura de educación física). Estos materiales dirigidos a la familia juegan un papel crucial puesto que permiten hacer partícipe a madres y padres de los propósitos generales de la asignatura y del proceso de enseñanza-aprendizaje en el que participan sus hijas e hijos. En este sentido, la formación del profesorado debería hacerse eco de la necesidad de sensibilizar a los futuros docentes y al profesorado en activo en la importancia de utilizar materiales para la familia. Reconocemos que no es tarea fácil puesto que requiere saber identificar aquellas cuestiones que se consideren importantes para compartir con las familias, así como los medios para poder favorecer la comunicación entre ambos.

Movimento, Porto Alegre, v. 10, n. 1, p. 41-70,janeiro/abril de 2004 


\section{Comentarios finales}

Los materiales curriculares impresos son una pieza más del desarrollo curricular de la educación física que debe estar en coherencia con el resto de elementos curriculares (p. ej. estrategias metodológicas y contenidos). Su relevancia para la formación del profesorado deriva de su naturaleza y utilidad porque es una herramienta muy característica del trabajo docente del profesorado con la cual debe familiarizarse el estudiante que quiere ser docente y con la cual debe comprometerse el profesorado en activo para mejorar su trabajo cotidiano.

Desde nuestro punto de vista, la formación inicial debe propiciar el camino para que la intervención de los futuros docentes en las tareas curriculares sea cada vez más autónoma. Por ello, consideramos necesario incorporar en los programas de formación contenidos relacionados con el análisis, la elaboración, el uso y la evaluación de materiales curriculares. Tal y como señala Cebrián de la Serna (1993: 233), es necesaria una formación para la autoproducción, es decir, "para saber diseñar y evaluar los materiales curriculares, desde donde se crean hábitos y modos de reflexión sobre los elementos psicológicos, comunicativos, ideológicos, sociopolíticos que todo material didáctico implica".

La elaboración de materiales por parte de los estudiantes no debe considerarse una meta sino una trayectoria de perfeccionamiento hacia la autonomía y emancipación de los futuros docentes. No obstante, si bien la experiencia de implicarse en este proceso suele resultar muy motivadora y enriquecedora para ellos, el profesorado debería ayudarles a identificar las posibilidades y limitaciones de sus conocimientos y habilidades, a aprender del análisis de los materiales publicados y a crear otros nuevos.

Del mismo modo, los programas de formación inicial y permanente deben favorecer las destrezas de cooperación y colaboración, así como los climas de trabajo en grupo en los que se 'comparte' el conocimiento y las experiencias sobre los materiales y la enseñanza de la educación física. De esta manera, se espera que la modificación, adaptación y/o creación de materiales curriculares no sólo se ajuste a los contextos y necesidades particulares del profesorado, sino también se conviertan en aspectos importantes del desarrollo profesional de los docentes.

Movimento, Porto Alegre, v. 10, n. 1, p. 41-70,janeiro/abril de 2004 


\section{Agradecimientos}

El estudio que proporciona los datos empíricos a este trabajo ha sido subvencionado con una ayuda recibida por la Universitat de Valencia a proyectos de investigación del año 2000-2001.

Um estudo sobre os textos impressos em educação física: implicações para a formação de professores Resumo: Neste artigo apresenta-se a fase descritiva de uma investigação sobre os materiais curriculares impressos derivados da análise de um questionário elaborado para esse fim. O questionário foi aplicado sob a forma de entrevista em uma amostra representativa de professores e professoras de educação física da Província de Valencia que trabalham no ensino secundário obrigatório. Apresentam-se os resultados gerais relativos à escolha, a elaboração e o uso desses materiais. Posteriormente, discutem-se esses resultados e se comenta suas implicações para a formação do professorado. Palavras-chave: Materiais Curriculares, Educação Física, Pensamento do Professorado, Espanha, Formação do Professorado.

A study of the printed texts in physical education: implications for the formation of teachers Abstract: In this paper, the descriptive phase of a research on printed curriculum materials derived from the analysis of a questionnaire is presented. This questionnaire is applied to a representative sample of physical education teachers that belong to the Valencian Autonomy and teach in Secondary Compulsory Education. General results concerning to selection, elaboration and use of such materials are presented. Finally, results are discussed and its implications in teacher education are also commented. Keywords: Curriculum Materials, Physical Education, TeacherThinking, Spain, Teacher Education

Movimento, Porto Alegre, v. 10, n. 1, p. 41-70,janeiro/abril de 2004 


\section{Referencias}

ABRAHAM, M. y ROJAS, A. La investigación educativa en Latinoamérica. Revista de Educación, 312, 21-42, 1997

ANYON, J. Social class and school knowledge. Curriculum Inquiry, 11 (1): 3-42. 1981.

APPLE, M.W. Curricular form and the logic of technical control: building the possessive individual. En: M.W. APPLE (ed.) Cultural and economic reproduction in education: Essays on class, ideology and the State, Londres: Routledge \& Kegan Paul, 1982. 247-274.

APPLE, M.W. Economía política de las publicaciones de libros de texto. Revista de Educación, 275, 43-62, 1984.

APPLE, M.W. Maestros y textos. Una economía política de las relaciones de clase y de sexo en educación. Barcelona: Paidós/MEC. 1989.

AREA, M. Un estudio sobre las decisiones docentes de uso del libro de texto en situaciones de enseñanza. En: L.M. VILLAR (ed.) Pensamientos de los profesores y toma de decisiones. Sevilla: Servicio de Publicaciones de la Universidad de Sevilla, 1986. 422435.

AREA, M. Los medios, los profesores y el currículo. Hospitalet de Llobregat: Sendai. 1991.

AREA, M. Los medios y materiales impresos en el curriculum. En: J.Ma Sancho (coord.) Para una tecnología educativa, Barcelona: Horsori, 1991. 85-113.

ARGIBAY, M.; CELORIO, G. y CELORIO, J.J. Análisis de los libros de texto de Ciencias Sociales. Una visión desde la educación para el desarrollo. Colección Documentos 2. Valencia: Generalitat Valenciana. 1990.

BEN-PERETZ, M. y TAMIR, $R$ What Teachers Want to Know about Curriculum Materials. Journal of Curriculum Studies, 13 (1), 45-54. 1981

BLANCO, N. Mujeres y hombres para el siglo XXI: El sexismo en los libros de texto. En: M.A. Santos (coord.) El harén pedagógico. Perspectiva de género en la organización escolar. Barcelona: Graó. 2000.

BROWNE, J. Gender bias in physical education textbooks. ACHPER National Journal, 127, 4-7. 1990

CALVO, T Los racistas son los otros. Gitanos, minorías y derechos humanos en los textos escolares. Madrid: Editorial Popular. 1989.

CANTARERO, J.E. Materiales curriculares y descualificación docente. Análisis interpretativo de las estrategias a través de las que el libro de texto regula el trabajo del profesorado. Tesis doctoral. Universitat de Valencia. 2000.

CLARK, R.E. Reconsidering research on Learning from media. Review of Educationa Research, 54 (4), 445-460. 1983.

CEBRIAN DE LA SERNA, M. La formación permanente del profesorado desde la autoproducción conjunta de los materiales didácticos. Una propuesta práctica. Qurrículum, $6-7,227-240,1993$

Movimento, Porto Alegre, v. 10, n. 1, p. 41-70,janeiro/abril de 2004 
CONNELLY, F.M. y BEN-PERETZ, M. Teachers' roles in the using and doing of research and curriculum development. Journal of Curriculum Studies, 12, 95-107. 1980.

DELGADO, M.A. BARRERA, J. y MEDINA, J. Análisis del libro de texto en la enseñanza de la educación física. Habilidad Motriz, 1, 11-18. 1992.

DEVÍS, J. Educación física, deporte y curriculum. Investigación y desarrollo curricular. Madrid: Visor. 1996.

DEVÍS, J. y PEIRÓ, C. Los materiales curriculares en la educación física. En: A. FRAILE (coord.) Manual de didáctica de la educación física. Madrid: Biblioteca Nueva. 2004.

DEVÍS, J.; PEIRÓ, C; MOLINA, J.R; VILLAMÓN, M.; ANTOLÍN, L y RODA, F LoS materiales curriculares impresos en educación física: clasificación, usos e investigación. Movimento, 15, 119-136. 2001.

DEWAR, A. Curriculum development and teachers' work: the case of the Basic Stuff Series in physical education. En: M. CARNES (ed.) Proceedings of the Fourth Conference on Curriculum Theory in Physical Education, Atenas: University of Georgia, 1985. 158-167.

FOX, D.J. El proceso de investigación en Educación. Pamplona: Eunsa. 1981.

FRAILE, A. (2000) Factores obstaculizadores y facilitadores en la formación del profesorado de educación física. En O.R. CONTRERAS (coord.) La formación inicial y permanente del profesor de educación física (Vol.II), Cuenca: Ediciones de la Universidad Castilla-La Mancha, 2000. 95-108.

FULLAN, M. Evaluating program implementation: What can be learned from Followthrough. Curriculum Inquiry, 13, 215-227. 1983.

GARCIA PASCUAL, E. Libros de texto y reforma educativa: Un análisis de los textos escolares de educación primaria. Tesis doctoral. Universidad de Zaragoza. 1996.

GAR RETA, N. y CAREAGA, R Modelos masculino y femenino en los textos de EGB. Madrid: Ministerio de Cultura: Instituto de la Mujer. 1987.

GIMENO, J. Los materiales y la enseñanza. Cuadernos de Pedagogía, 194, 10-15. 1991.

GÜEMES, R. Libros de texto y desarrollo del currículo en el aula. Un estudio de casos. Tesis doctoral. Universidad de La Laguna. 1994.

HANKE, U. Cognitive aspects of interaction in physical education. En G. BARRETTE, R. FEINDGOLD, C. REES y M. PIERON (eds.) Myths, Models and Methods, Champaign: Human Kinetics, 1987.135-141.

JONHSEN, E.B. Libros de texto en el calidoscopio. Barcelona: Pomares. 1996.

KIRK, D. School knowledge and the curriculum package-as-text. Journal of Curriculum Studies, 22, 409-425. 1990.

LOEWENBERG, D. y FEIMAN-NEMSER, S. Using textbooks and teachers' guides: a dilema for beginning teachers and teacher educators. Curriculum Inquiry, 18 (4): 401-423. 1988

Movimento, Porto Alegre, v. 10, n. 1, p. 41-70,janeiro/abril de 2004 
LÓPEZ, R. y de la CABA, M.A. Formación sociopersonal del ciudadano en los libros de texto de conocimiento del medio (segundo ciclo de primaria). Bordón. Revista de Pedagogía, 54 (1), 69-82. 2002.

MARSH, C.J. Teachers' Knowledge of and Use of Social Studies Curriculum Materials in Public Elementary Schools'. Journal of Educational Research, 77 (4), 237-243. 1984.

MARSH, C.J.; WILLIS, S.; DESCHAMP, R y DAVIS, B.R Teachers Perceptions about the Selection, Distribution and Use of Social Studies and Mathematics Curriculum Materials within a State Education System. Journal of Curriculum Studies, 17 (1), 49-61. 1985.

MARTINEZ BONAFÉ, J. Trabajar en la escuela. Profesorado y reformas en el umbral del siglo XXI. Madrid: Miño y Dávila Editores. 1998.

MOLINA, J.R; DEVÍS, J.; MARTíNEZ, R y SOLER, D. Proceso de elaboración de un cuestionario para el estudio del uso de los materiales curriculares impresos en Educación Física. En: V. MAZÓN et al. (coord.) La enseñanza de la Educación Física y el Deporte Escolar. Actas del IV Congreso Internacional, Santander: ADEF Cantabria, 2001a. 688694.

MOLINA, J.R; DEVÍS, J.; MARTÍNEZ, R y SOLER, D. Características de un cuestionario para el estudio del uso de los materiales curriculares impresos en Educación Física. En: J.F. CAMPOS, S.LLANA y R. ARANDA (coord.) Nuevas aportaciones al estudio de la actividad física y el deporte. II Congreso de Ciencias de la Actividad Física y el Deporte. FCCAFE-Universitat de Valencia. Valencia, 2001b. 1027-1037.

MORENO, A. El arquetipo viril protagonista de la historia. Barcelona: La Sal.. 1987.

NÍAS, J. Learning from Difference: A Collegial Approach to Change. En J. SMYTH (ed.) Educating Teachers. Changing the Nature of Pedagogical Knowledge. Londres: The Falmer Press, 1987. 137-152.

PARCERISA Els materials curriculars a l'ensenyament Primari. Aná/isi dels materials curriculars editats en suport paper uti/itzats a l'Educado Primaria del nou sistema educatiu. Tesis doctoral. Universitat de Barcelona. 1995.

PARCERISA, A. Materiales curriculares. Cómo elaborarlos, seleccionarlos y usarlos. Barcelona: Graó. 1996

PEIRÓ, C. Materiales curriculares y formación del profesorado en educación física. Tándem. Didáctica de la Educación Física (Número monográfico sobre materiales curriculares), 4, 19-33. 2001.

PEIRÓ, C. y DEVÍS, J. El análisis de materiales curriculares en educación física: un ejemplo. En: L MONTERO y J.M. VEZ (eds.) Las didácticas específicas en la formación del profesorado II (vol. II), Santiago de Compostela: Tórculo Edicions, 1994. 775-781.

PEIRÓ, C. y DEVíS, J. Health-Based Physical Education in Spain: The Conception, Implementation and Evaluation of an Innovation. European Physical Education Review, 1(1), 37-54. 1995

PLACEK, J. An evaluation of the implementation of Basic Stuff. Journal of Teaching in Physical Education, 8, 152-161. 1989.

Movimento, Porto Alegre, v. 10, n. 1, p. 41-70,janeiro/abril de 2004 
PUTNAM, R. T Teaching the 'hows' of mathmatics for everyday life: A case of fiftht-grade teacher's interpretation of policy. Elementary School Journal, 93 (2), 163-177. 1992.

REMILLARD, J.T. Teaching mathematics for understanding: A fifht-grade teacher's interpretation of policy, Elementary School Journal, 93(2), 179-193. 1992.

REMILLARD, J.T Curriculum Materials in Mathematics Education Reform: A Framework for Examining Teachers' Curriculum Developement, Curriculum Inquiry, 29 (3), 315-342. 1999.

REMILLARD, J. Can curriculum materials support teachers' learning? Two fourth-grade teachers' use of a new mathematics text. The Elementary School Journal, 100 (4), 331 350. 2000.

RIBAS, A. y GUERRERO, J.A. Análisis del sexismo en libros de texto en educación física. En: R CLIMENT; C. BLASCO y Ma J. LÓPEZ (coords.) La enseñanza de la educación física, Valencia: Institut Valencia de la Dona -Generalitat Valenciana, 1994. 379-390.

RODRígueZ DiÉGUEZ, J.L. et al. Planes de Estudio y Métodos de Enseñanza (3). Diagnósfico del Sistema Educativo. 1997. (La Escuela Secundaria Obligatoria). MEC. Madrid. 1998.

SAN MARTíN, A. La organización escolar. Cuadernos de Pedagogía, n. 194, 26-28. 1991. SANTOS, M.A. ¿Cómo evaluar los materiales? Cuadernos de Pedagogía, n. 194, 29-31. 1991.

SHANNON, $\mathrm{R}$ The use of commercial reading materials in American elementary schools. Reading Research Quarterly, 19 (1), 68-85. 1983.

SCHEMPP, R A critical analysis of the implications in Basic Stuff. Journal of Physical Education and Recreation, 53, 20-22. 1982.

STODOLSKY, S. S. Is teaching really by the books. En: Ph. Jackson (ed.): From Socrates to Software: The Teacher as Text and the Text as a Teacher. Eightieth Yearbook of the National Study of Education, University of Chicago Press, 1989. 159-184.

TIANA, A. El libro escolar como instrumento didáctico. Concepciones, usos e investigaciones, en A. ESCOLANO (dir.) Historia ilustrada del libro escolar en España. De la posguerra a la reforma educativa, Madrid: Fundación Germán Sánchez Ruipérez, 1998. 149-175.

TINNING, R. Educación física: la escuela y sus profesores. Valencia: Servei de Publicacions de la Universitat de Valencia. 1992.

VENEZKY, R.L. Textbooks in school and society. En: RW. JACKSON (ed.) Handbook of research on curriculum, Nueva York: Macmillan Pub. Comp., 1992. 436-461.

WESTBURY, I. Textbooks, Textbooks Publishers and the Quality of Schooling. En: A. WOODWARD y D.L. ELLIOT (eds.) NSSE Yearbook 1990: Part I. Textbooks and Schooling in the United States, University of Chicago Press. 1990

Movimento, Porto Alegre, v. 10, n. 1, p. 41-70,janeiro/abril de 2004 
WOODWARD, A., ELLIOT, D.L. y NAGEL, K.C. Textbooks in school and society: an annotated bibliography and guide to research. Nueva York: Garland. 1988.

ZAHORIK, J.A. Teaching Style and Textbooks, Teaching and Teacher Education, 7 (2), 185-196. 1991.

ZABALA, M.A. Materiales curriculares. En: T MAURI et al. (coords.) El curriculum en el centro educativo. Barcelona: ICE de la Universitat de Barce-Iona-Horsori 1990. 125-167.

José Devís Devís

Departament d'Educació Física i Esportiva Facultat de Ciències de l'Activitat Física i l'Esport C/ Gascó Oliag, 3 46010 Valencia (España)

jose.devis@uv.es

Movimento, Porto Alegre, v. 10, n. 1, p. 41-70,janeiro/abril de 2004 